\title{
VARIACIONES EN LA REALIZACIÓN DE LA ESTRUCTURA ARGUMENTAL PREFERIDA EN NIÑOS Y ADULTOS A PARTIR DE LA NARRACIÓN DE ANÉCDOTAS PERSONALES Y CUENTOS EN IMÁGENES*
}

\section{VARIATIONS IN THE REALIZATION OF THE PREFERRED ARGUMENT STRUCTURE IN CHILDREN AND ADULTS FROM TWO ELICITATION TASKS}

Carolina Castillo Hernández

Universidad Autónoma de Querétaro, Facultad de Lenguas y Letras

Valeria A. Belloro

Universidad Autónoma de Querétaro, Facultad de Lenguas y Letras

Luisa Josefina Alarcón Neve

Universidad Autónoma de Querétaro, Facultad de Lenguas y Letras

\section{RESUMEN}

En este trabajo se exponen los resultados del análisis de narrativas de adultos y de niños bajo la perspectiva de la estructura argumental preferida (EAP; Du Bois, 1987). El corpus se conformó con base en dos tareas de elicitación: la narración de un cuento a partir de imágenes y la narración de una anécdota personal. Los resultados muestran que las tendencias de la EAP son similares en niños y adultos, pero con cambios significativos entre las dos tareas de elicitación. En particular, un segundo análisis de los datos muestra una preferencia relativa, en la tarea del cuento, por la codificación léxica de tópicos continuos, lo que sugiere que los hablantes (y particularmente los niños) combinan, para esta tarea específica, estrategias narrativas con estrategias de descripción de imágenes independientes.

* Agradecemos las cuidadosas observaciones de dos dictaminadores anónimos, que nos permitieron clarificar los objetivos y enriquecer nuestro análisis. Cualquier deficiencia remanente es nuestra responsabilidad. Este trabajo se realizó en el marco del proyecto Aspectos de la interfaz sintaxis-pragmática en español y lenguas de México (Conacyt 256785), a cargo de Valeria A. Belloro. 
PAlABRAS ClAVE: tendencias discursivas; discurso infantil; continuidad tópica; interfaz sintaxis-pragmática

\section{Abstract}

In this paper we present the results from an analysis of narratives by adults and children in terms of their Preferred Argument Structure (PAS; Du Bois, 1987). The corpus was built from two elicitation tasks: telling a story from pictures and narrating a personal experience. Our results show that PAS trends in children and adults are similar, but with significant differences between the two elicitation tasks. A second analysis of the data shows a relative preference for using a noun phrase to refer to continuous topics in the storytelling. This suggests that, for this particular task, speakers (and particularly children) combine narrative strategies with strategies used for describing independent images.

KEYWORDS: discursive trends; child discourse; topic continuity; Syntax-Pragmatics Interface 
Variaciones en la realización de la estructura argumental preferida en niños y adultos 39

\section{INTRODUCCIÓN}

El desarrollo discursivo se inicia a edades muy tempranas - alrededor de los dos años-, y evoluciona durante la etapa escolar básica del niño (Barriga, 2002). En sus primeros intentos de crear un discurso narrativo, el niño construye la función referencial, la cual se conforma de reportes fragmentados que son complementados con las preguntas que le hace el interlocutor (Romero \& Gómez, 2013: 27). En este desarrollo de habilidades discursivas los niños deben familiarizarse con las características del discurso narrativo, tales como hablar del "ahí y entonces", tomar turnos más largos y organizar la narración para mantener la atención del interlocutor durante el relato (Shiro, 2007, 2012). Alrededor de los ocho años de edad, los niños comienzan a construir la función expresiva (Barriga, 2002). Es en estos primeros años de la educación primaria cuando el discurso narrativo de los niños muestra avances en su coherencia y cohesión, y es en esta etapa en la que nos enfocaremos en el presente estudio. En particular, nuestro interés es evaluar, a partir del modelo de la estructura argumental preferida (EAP; Du Bois, 1987; Du Bois, Kumpf \& Ashby, 2003), en qué medida el desarrollo de capacidades discursivas de los niños de 8 años aparece reflejado también en el tipo de codificación de los argumentos verbales de acuerdo con su función, aspecto estudiado ampliamente para el discurso adulto.

Si bien existe ya una abundante literatura sobre el desarrollo de las competencias narrativas en los niños hispanohablantes ( $c f$. Berman \& Slobin, 1994; Shiro, 1999, 2007, 2012; Kupersmitt \& Berman, 2001; Barriga, 2002, 2014; Aguilar, 2003; Hess, 2010; Hess \& Auza, 2013), la perspectiva que ofrece el modelo de la estructura argumental preferida ha sido relativamente menos estudiada (pero véase Amaro, 2010). La EAP analiza la distribución de la información en el discurso, centrándose en el tipo de realización y estatus pragmático de los argumentos centrales directos de los predicados: A, si se trata de un sujeto de verbo transitivo; S, si es un sujeto de verbo intransitivo, y $\mathrm{O}$, si es el objeto directo de un verbo transitivo. Así, se ha demostrado que, en la organización del discurso natural, hay estructuras y alineaciones preferidas (más frecuentes) y sancionadas (menos frecuentes). Estas generalizaciones se capturan bajo la forma de cuatro máximas o tendencias que se muestran en el Cuadro 1. 
40 Carolina Castillo Hernández, Valeria A. Belloro \& Luisa Josefina Alarcón Neve

CuADRo 1. Tendencias de la estructura argumental preferida (adaptado de Du Bois, 1987)

Gramática

CAntidAD Evita más de un argumento léxico por cláusula

Evita que los argumentos léxicos aparezcan en el rol de $A$
PragmáticA

Evita más de un argumento nuevo por cláusula

Evita que los argumentos nuevos aparezcan en el rol de A

La primera tendencia, Evita más de un argumento léxico por cláusula, captura el hecho de que, si se consideran las cláusulas transitivas en un discurso natural, la mayoría de ellas tiene, como máximo, solo una de sus posiciones argumentales $(\mathrm{A}, \mathrm{O})$ realizadas por una frase nominal con núcleo sustantivo $(i . e$. por un argumento léxico). La segunda tendencia, Evita más de un argumento nuevo por cláusula, analiza la denotación de los argumentos A, O para capturar que, en las cláusulas transitivas de un discurso natural, son frecuentes los casos en los que tanto A como $\mathrm{O}$ codifican referentes ya mencionados en el discurso, o bien aquellas cláusulas en las cuales solo uno de estos dos argumentos denota un referente discursivo que se menciona por primera vez (i. e. nuevo). Es decir, estas dos primeras tendencias capturan que en el discurso natural son más frecuentes las estructuras del tipo de (1a), en las que aparece como máximo un argumento léxico, que las del tipo de (1b), donde los dos argumentos transitivos son léxicos. Asimismo, son más frecuentes las estructuras del tipo de (2a), donde aparece como máximo un argumento nuevo, que las del tipo de (2b), donde los dos argumentos son nuevos. ${ }^{1}$

(1) a. Él la encontró / Él encontró la rana.

b. El niño encontró la rana.

(2) a. Él la encontró / Él encontró una rana.

b. Un niño encontró una rana.

La tercera tendencia, Evita que los argumentos léxicos aparezcan en el rol de $A$, captura el hecho de que, considerando todos los $\mathrm{A}, \mathrm{S}$ y $\mathrm{O}$ de un discurso na-

1 La relación biunívoca entre estatus pragmático y definitud es una simplificación que nos permitimos aquí con fines meramente ilustrativos. 
tural, la mayoría de los que se realizan como léxicos se alinearán con los roles de $\mathrm{S}$ y $\mathrm{O}$, a exclusión de A. Finalmente, la última tendencia, Evita que los argumentos nuevos aparezcan en el rol de A captura que, considerando todos los A, $\mathrm{S}$ y $\mathrm{O}$ de un discurso natural que denotan referentes discursivos nuevos, la mayoría se alineará con los roles de $\mathrm{S}$ y $\mathrm{O}$, a exclusión de A. Como es evidente, estas dos últimas tendencias no comparan la frecuencia relativa de distintos tipos de cláusulas (como las de (1) y (2) arriba) sino la distribución por rol de todos los argumentos léxicos o nuevos de un texto. ${ }^{2}$

La presencia de estas cuatro tendencias se ha validado en distintas lenguas, así como en distintos géneros discursivos, tanto en adultos (Du Bois, 1987; Bentivoglio, 1992, 1994; Ashby \& Bentivoglio, 1993; Du Bois, Kumpf \& Ashby, 2003, entre otros) como en niños (Clancy, 1993, 1995, 1996, 1997, 2003; Bentivoglio, julio, 1996; Rubino, 1996; Allen \& Schröder, 2003; Khorounjaia \& Tolchinsky, 2004). Este trabajo busca contribuir a las observaciones del discurso narrativo infantil describiendo en qué medida este se asemeja o diferencia del discurso narrativo adulto en términos del modelo de la EAP, y mostrando el tipo de estrategias con las que se mantiene la referencia a los personajes dentro del discurso narrativo en ambas poblaciones.

\section{CORPUS Y METODOLOGÍA}

El corpus analizado consta de narraciones orales de niños de 8 años y de adultos de entre 18 y 25 años de edad, a partir de dos tareas de elicitación. La primera, a la que nos referiremos como corpus de anécdotas, se obtuvo a partir de las preguntas ¿Cuál ha sido el peor día de tu vida? ¿Cuál fue el día en que más te asustaste?, inspiradas en el trabajo de Labov (1972), y está compuesto por 12 narrativas infantiles y 6 de adultos. Estos datos se compararon con los de la segunda tarea, a la que nos referiremos como corpus de cuentos, que se conformó solicitando a los participantes la narración del cuento en imágenes One frog too many (Mayer \& Mayer, 1977), compuesto por 12 narrativas infantiles y 8 de adultos. Las anécdotas infantiles y 7 cuentos de adultos se recogieron en el marco del proyecto Adjetivos y adverbios en cuentos generados por niños y

2 Para una presentación reciente y más detallada del modelo, así como algunas aplicaciones y extensiones, véase Belloro y Guerrero (2012). 
adolescentes en edad escolar, a cargo de Alarcón Neve (Universidad Autónoma de Querétaro, Registro FLL-2009-06). Las anécdotas de adultos, uno de los cuentos de adultos y los cuentos de niños fueron recogidos por Castillo para esta investigación. Los datos fueron segmentados en cláusulas siguiendo la propuesta de Berman y Slobin (1994: 657-664), considerando la cláusula como la unidad básica cuyo núcleo es un predicado unificado; esto es, un predicado que expresa una sola situación, ya sea actividad, evento o estado. Se recogieron, en total, más narrativas infantiles que de adultos con el objeto de obtener un número comparable de cláusulas en las dos poblaciones. El total de cláusulas que conforman cada corpus se presenta en el Cuadro 2.

CuAdRo 2. Conformación del corpus por tipo de cláusula

\begin{tabular}{|c|c|c|c|c|c|}
\hline & \multicolumn{2}{|c|}{ Cuentos } & \multicolumn{2}{|c|}{ AnÉCDOTAS } & \multirow{2}{*}{ Totales } \\
\hline & Transitivas & Intransitivas & Transitivas & Intransitivas & \\
\hline Niños & 178 & 312 & 24 & 60 & 574 \\
\hline Adultos & 181 & 301 & 31 & 59 & 572 \\
\hline Totales & 359 & 613 & 55 & 119 & 1146 \\
\hline
\end{tabular}

Nota: El desglosado en términos de transitividad responde a que las cláusulas transitivas solo son relevantes para dos de las tendencias.

Siguiendo la metodología de la EAP, además de distinguir entre cláusulas transitivas e intransitivas, se etiquetaron los argumentos centrales directos de cada cláusula de acuerdo con su función (A, S u O), su estatus pragmático (dado o nuevo) y su realización (léxicos o no léxicos). Ya fuera del marco de la EAP - y con el fin de analizar más en detalle el inesperado uso de frases nominales en la codificación de tópicos continuos (Givón, 1983), que aparecía fundamentalmente en el corpus de cuentos narrados por niños-, se consideró por separado los distintos tipos de expresiones referenciales léxicas con base en los trabajos de la escuela givoniana de continuidad tópica (Givón, 1983; Bentivoglio, 1983). Para este propósito se consideró el total de argumentos centrales directos de cada corpus, cuyo desglose global se presenta en el Cuadro 3.

En lo que sigue, presentaremos primero los resultados en términos de las predicciones del modelo de la EAP, para luego enfocarnos brevemente en los contextos de uso de los distintos tipos de expresiones referenciales que surgen de la comparación entre tareas. 
Variaciones en la realización de la estructura argumental preferida en niños y adultos 43

CUADRO 3. Distribución de argumentos centrales directos

\begin{tabular}{|c|c|c|c|c|c|c|c|}
\hline & \multicolumn{3}{|c|}{ Cuentos } & \multicolumn{3}{|c|}{ AnÉCDOTAS } & \multirow{2}{*}{ Totales } \\
\hline & $S$ & $A$ & 0 & $S$ & A & 0 & \\
\hline Niños & 312 & 178 & 178 & 60 & 24 & 24 & 776 \\
\hline Adultos & 301 & 181 & 181 & 59 & 31 & 31 & 784 \\
\hline Totales & 613 & 359 & 359 & 119 & 55 & 55 & 1560 \\
\hline
\end{tabular}

\section{LA ESTRUCTURA ARGUMENTAL PREFERIDA EN DOS CORPUS NARRATIVOS} INFANTILES

\subsection{Evita más de un argumento léxico por cláusula}

Como señalamos en la introducción, de acuerdo con esta tendencia se espera que, dentro de las cláusulas transitivas, sean frecuentes las que codifican sus argumentos en forma exclusivamente pronominal (3a), o las que a lo sumo realizan uno de ellos a través de una frase léxica (3b). Por el contrario, serán infrecuentes, en comparación, las cláusulas transitivas con dos argumentos léxicos (3c).

(3) a. Ellos no la encontraron. [Christian, cuento 6]

b. Él tenía tres mascotas. [Christian, cuento 6]

c. Un niño recibió un regalo. [Alejandro, cuento 8]

Los resultados cuantitativos respecto de esta tendencia se presentan en el Cuadro 4 para el corpus de anécdotas y el Cuadro 5 para el corpus de cuentos. En la primera columna se incluyen aquellas cláusulas en las que sus dos argumentos son expresados de forma exclusivamente pronominal. En la segunda se contabilizan las cláusulas donde uno de los argumentos transitivos, ya sea $\mathrm{A} \mathrm{u} \mathrm{O}$, se realizó de forma léxica. En la tercera se incluyeron las cláusulas en las que tanto el argumento A como el $\mathrm{O}$ se codificaron léxicamente. 
44 Carolina Castillo Hernández, Valeria A. Belloro \& Luisa Josefina Alarcón Neve

CuADRo 4. Número de argumentos léxicos por cláusula en el corpus de anécdotas con respecto a la tendencia Evita más de un argumento léxico

\begin{tabular}{|c|c|c|c|c|c|c|c|}
\hline & \multicolumn{2}{|c|}{0 ARG. LÉX. } & \multicolumn{2}{|c|}{1 ARG. LÉX. } & \multicolumn{2}{|c|}{2 ARG. LÉX. } & \multirow{2}{*}{ Tota } \\
\hline & $\#$ & $\%$ & \# & $\%$ & $\#$ & $\%$ & \\
\hline Niños & 6 & 25 & 16 & 67 & 2 & 8 & 24 \\
\hline Adultos & 11 & 35 & 17 & 55 & 3 & 10 & 31 \\
\hline
\end{tabular}

CuAdro 5. Número de argumentos léxicos por cláusula en el corpus de cuentos con respecto a la tendencia Evita más de un argumento léxico

\begin{tabular}{|c|c|c|c|c|c|c|c|}
\hline & \multicolumn{2}{|c|}{0 ARG. LÉX. } & \multicolumn{2}{|c|}{1 ARG. LÉX. } & \multicolumn{2}{|c|}{2 ARG. LÉX. } & \multirow{2}{*}{ Total } \\
\hline & $\#$ & $\%$ & $\#$ & $\%$ & $\#$ & $\%$ & \\
\hline Niños & 43 & 24 & 77 & 43 & 58 & 33 & 178 \\
\hline Adultos & 37 & 20 & 84 & 46 & 60 & 33 & 181 \\
\hline
\end{tabular}

Los datos muestran una frecuencia mayor considerable de cláusulas con dos argumentos léxicos en el corpus de cuentos $(33 \%)$ con respecto al de anécdotas (8 y $10 \%$ para niños y adultos, respectivamente). Volveremos sobre esta cuestión abajo (§3.5). Por lo pronto, sin embargo, es interesante notar también que no aparecen diferencias significativas entre las dos poblaciones, mostrando niños y adultos un comportamiento discursivo similar, que se adhiere a las predicciones generales de la EAP. Es decir, tanto niños como adultos prefieren, en ambas tareas, las cláusulas transitivas con un argumento léxico como máximo, y los casos de cláusulas transitivas con dos argumentos léxicos son, como se espera, relativamente minoritarios.

\subsection{Evita argumentos léxicos en el rol de A}

Esta tendencia predice que, del total de argumentos léxicos presentes en el discurso, la mayoría se relacionará con los roles de $\mathrm{S}$ y $\mathrm{O}$, evitando el rol de $\mathrm{A}$. Recordemos que aquí no se cuenta el número de cláusulas sino el de argumentos léxicos. Sin embargo, simplificando solo con fines ilustrativos, son más acordes con esta tendencia las estructuras del tipo de (4a) y (4b), donde el argumento léxico se coloca en el rol de $\mathrm{S}$ u O, que las del tipo de (4c) en las que el argumento léxico se coloca en función de A. 
Variaciones en la realización de la estructura argumental preferida en niños y adultos 45

(4) a. Y entonces el perro ladró. [Luis, cuento 11]

b. ...encontrar un taxi o un teléfono. [Guillermo, anécdota 5]

c. Sus papás le dieron un regalo. [Diana, cuento 3]

Como se observa en el Cuadro 3 arriba, en las anécdotas de niños ocurren 108 posiciones argumentales centrales directas (A, S, O) y en las de adultos, 121. De estas posiciones argumentales, 34 están realizadas de forma léxica entre los niños y 36 entre los adultos. La distribución de estos argumentos léxicos se presenta en el Cuadro 6. En cuanto al corpus de cuentos, se contabilizaron 668 posiciones argumentales para el corpus infantil y 663 para el de adultos (véase el Cuadro 3). De estas, solo 396 y 369, respectivamente, se realizaron de forma léxica, como se aprecia en el Cuadro 7.

CuADRo 6. Distribución de argumentos léxicos por rol gramatical en el corpus de anécdotas con respecto a la tendencia Evita A léxicos

\begin{tabular}{|c|c|c|c|c|c|}
\hline & \multicolumn{2}{|c|}{ ARG. LÉX. COMO S/0 } & \multicolumn{2}{|c|}{ ARG. LÉX. COMO A } & \multirow{2}{*}{ Total } \\
\hline & $\#$ & $\%$ & \# & $\%$ & \\
\hline Niños & 28 & 82 & 6 & 18 & 34 \\
\hline Adultos & 32 & 89 & 4 & 11 & 36 \\
\hline
\end{tabular}

Cuadro 7. Distribución de argumentos léxicos por rol gramatical en el corpus de cuentos con respecto a la tendencia Evita A léxicos

\begin{tabular}{|c|c|c|c|c|c|}
\hline & \multicolumn{2}{|c|}{ ARG. LÉX. COMO S/0 } & \multicolumn{2}{|c|}{ ARG. LÉX. COMO A } & \multirow{2}{*}{ Total } \\
\hline & \# & $\%$ & \# & $\%$ & \\
\hline Niños & 318 & 80 & 78 & 20 & 396 \\
\hline Adultos & 296 & 80 & 73 & 20 & 369 \\
\hline
\end{tabular}

Los datos muestran que, tal como se espera, los argumentos léxicos se ubican preferentemente en los roles de $\mathrm{S}$ y $\mathrm{O}$, evitando el rol de $\mathrm{A}$, en ambas poblaciones, con la salvedad, en este caso, de que en el corpus de anécdotas los niños ubican más argumentos léxicos en el rol de A que los adultos (18 vs. 11\%), aspecto sobre el que volveremos en $\$ 3.4$, al analizar cuántos de estos argumentos léxicos denotan participantes nuevos y de qué tipo. 


\subsection{Evita más de un argumento central nuevo}

Esta tendencia representa la contraparte pragmática de la tendencia a evitar cláusulas transitivas con más de un argumento léxico. Es necesaria en la medida en que ambas tendencias no necesariamente coinciden. En efecto, a menudo aparecen, en el discurso natural, codificaciones léxicas de argumentos que ya han sido introducidos. De acuerdo con esta tendencia, serán más frecuentes las cláusulas transitivas en las que ningún argumento es nuevo, o tienen solo un argumento nuevo, que las cláusulas con dos argumentos nuevos. Así, se prevé que serán más frecuentes las estructuras del tipo de (5a), donde los argumentos A y $\mathrm{O}$ son dados, y (5b), en la que solo uno de los argumentos es nuevo, que las del tipo de (5c), en la que tanto el argumento A como el $\mathrm{O}$ son nuevos. La distribución de los datos de acuerdo con esta tendencia se presenta en el Cuadro 8 para el corpus de anécdotas y el Cuadro 9 para el corpus de cuentos. Recuérdese que en relación con esta tendencia solamente son relevantes las cláusulas transitivas ( $c f$. Cuadro 2).

(5) a. Ø

b. Ø recibí un mensaje. [Alejandro, anécdota 2]

c. Un pequeño niño vio un hermoso regalo. [Dolores, cuento 2]

CuAdro 8. Número de argumentos nuevos por cláusula en el corpus de anécdotas con respecto a la tendencia Evita más de un argumento nuevo

\begin{tabular}{|c|c|c|c|c|c|c|c|}
\hline & \multicolumn{2}{|c|}{0 ARG. NVOS. } & \multicolumn{2}{|c|}{1 ARG. NVO. } & \multicolumn{2}{|c|}{2 ARG. NVOS. } & \multirow{2}{*}{ Total } \\
\hline & $\#$ & $\%$ & $\#$ & $\%$ & $\#$ & $\%$ & \\
\hline Niños & 8 & 33 & 14 & 58 & 2 & 8 & 24 \\
\hline Adultos & 13 & 42 & 16 & 52 & 2 & 6 & 31 \\
\hline
\end{tabular}

Cuadro 9. Número de argumentos nuevos por cláusula en el corpus de cuentos con respecto a la tendencia Evita más de un argumento nuevo

\begin{tabular}{|c|c|c|c|c|c|c|c|}
\hline & \multicolumn{2}{|c|}{0 ARG. NVOS. } & \multicolumn{2}{|c|}{1 ARG. NVO. } & \multicolumn{2}{|c|}{2 ARG. NVOS. } & \multirow{2}{*}{ Total } \\
\hline & $\#$ & $\%$ & \# & $\%$ & \# & $\%$ & \\
\hline Niños & 146 & 82 & 29 & 16 & 3 & 2 & 178 \\
\hline Adultos & 137 & 76 & 41 & 23 & 3 & 2 & 181 \\
\hline
\end{tabular}


Es interesante comparar los resultados de estos cuadros con los expuestos en el Cuadro 4 y el Cuadro 5 arriba. En el Cuadro 4 veíamos que, en el corpus infantil de anécdotas, aparecían 2 cláusulas transitivas con 2 argumentos léxicos. Esas 2 cláusulas son las mismas que en el Cuadro 8 aparecen con 2 argumentos nuevos. Es decir, en el corpus de anécdotas los niños emplearon cláusulas transitivas con 2 argumentos léxicos solamente en los casos en que debían codificar referentes discursivos nuevos. Una situación prácticamente idéntica resulta del corpus adulto: en el Cuadro 4 aparecían 3 cláusulas transitivas con 2 argumentos léxicos en las anécdotas narradas por adultos. En el Cuadro 8 vemos que 2 de esas cláusulas codifican referentes nuevos, y solo en una cláusula los adultos utilizaron codificaciones léxicas para referentes ya mencionados.

El panorama varía drásticamente si consideramos los cuentos. En el Cuadro 5 vimos que los niños produjeron 58 cláusulas transitivas con 2 argumentos léxicos y los adultos, 60. De ese total, solamente 3 cláusulas en cada población corresponden a argumentos nuevos (véase el Cuadro 9). De esto se deduce que, en el corpus de cuentos, los narradores, niños y adultos por igual, recurren sistemáticamente a codificaciones léxicas de referentes discursivos dados.

En muchos casos, la codificación léxica de referentes dados sirve para resolver contextos de ambigüedad referencial (\$3.5). La aparición de una segunda rana en el corpus cuentos crea frecuentemente tales contextos, y los niños de 8 años pueden detectarlos y resolverlos tan exitosamente como los adultos, ya sea creando distintos descriptores (rana vs. sapo), o complejizando la frase con modificadores que desambigüen la referencia (rana grande vs. rana chiquita):

(6) a. Ya en el camino la rana de una patada tiró al sapo. [Alonso, cuento 7]

b. Y la rana grande tiró la otra a la chiquita. [Francisco, cuento 2]

Esta es sin duda una de las motivaciones detrás del incremento de cláusulas transitivas con dos argumentos nuevos en los cuentos. Sin embargo, como veremos más adelante (§3.5), un análisis global de los contextos de uso de frases nominales en ambas poblaciones revela que el tipo de tarea cumple un rol importante, especialmente en la población infantil, que utiliza frases nominales también fuera de contextos de ambigüedad referencial. 


\subsection{Evita argumentos nuevos en el rol de A}

De acuerdo con esta tendencia esperamos que, del total de referentes nuevos que se introducen en el discurso en posiciones centrales directas, la mayoría se asocie con los roles de S y O, evitando el rol de A. Así, nuevamente simplificando la interpretación de la tendencia con fines ilustrativos, son más acordes con esta tendencia las estructuras del tipo de (7a), en donde el argumento nuevo se coloca en el rol de $\mathrm{S}$, y (7b), en la que el argumento nuevo se encuentra en función de $\mathrm{O}$, que las del tipo de (7c) donde el argumento nuevo está en el rol de A. Los resultados se presentan en el Cuadro 10 para el corpus de anécdotas y el Cuadro 11 para el corpus de cuentos.

(7) a. Alrededor de él se encontraba el perro Rufo, la rana Ojotes, la tortuga Eslugo. [Verónica, cuento 4]

b. Decirle la dirección. [Guillermo, anécdota 5]

c. Juanito el día de su cumpleaños recibió un regalo. [Alfonso, cuento 7]

CuAdRo 10. Rol gramatical de los referentes nuevos en el corpus de anécdotas con respecto a la tendencia Evita A nuevos

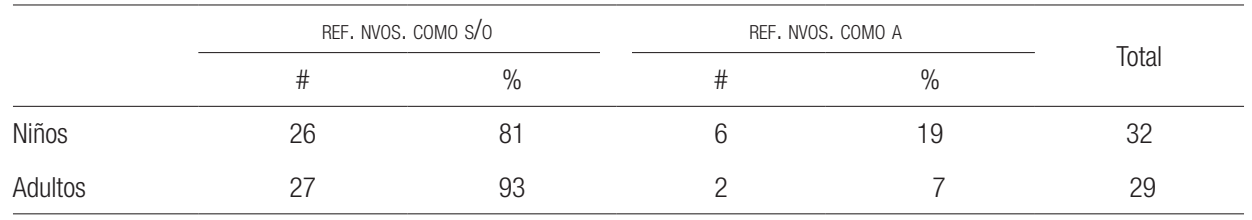

CuAdRo 11. Rol gramatical de los referentes nuevos en el corpus de cuentos con respecto a la tendencia Evita A nuevos

\begin{tabular}{lllllll}
\hline & \multicolumn{2}{c}{ REF. NVOS. COMO S/0 } & & \multicolumn{2}{c}{ REF. NVOS. COMO A } & Total \\
\cline { 2 - 3 } \cline { 5 - 7 } & $\#$ & $\%$ & & $\#$ & $\%$ & \\
\hline Niños & 53 & 93 & & 4 & 7 & 60 \\
Adultos & 62 & 94 & & 4 & 6 & 66 \\
\hline
\end{tabular}

En relación con esta tendencia vemos que también se cumple en lo general, y tanto niños como adultos ubican los referentes nuevos preferentemente en los roles de S/O en las dos tareas. Sin embargo, también notamos que, contrariamente a lo esperado, hay una mayor ocurrencia de argumentos nuevos en el rol de A en el corpus infantil de anécdotas (19\% frente a $7 \%$ de los adultos). Esto se 
relaciona con el también relativamente alto porcentaje de argumentos léxicos en el rol de A en el mismo sub-corpus (véase Cuadro 6). Es en relación con estas dos tendencias en este corpus específico donde notamos por primera vez que el discurso infantil se diferencia del adulto: los niños producen más argumentos léxicos y nuevos en el rol de A que los adultos, diferencia que surge solamente en el corpus de anécdotas. Al analizar estos ejemplos con más detalle, no obstante se observa que estos casos son del tipo de los presentados en (8).

(8) a. Mi tío me había asustado. [Pedro, anécdota 4]

b. Mi mamá me dejó en mi casa. [Facundo, anécdota 7]

c. Mi hermana la acababa de utilizar. [Tomás, anécdota 12]

La única diferencia del discurso infantil respecto del adulto, entonces, es que las anécdotas infantiles se construyen — cuestión poco sorprendente- alrededor del círculo familiar inmediato de los niños y que, con mayor frecuencia, son ellos los principales AGENTES de los eventos narrados. Así, es desde esta particularidad que se explica la relativa preeminencia de A nuevos — pero esperables- que se observa en los datos.

\subsection{Algunas observaciones sobre la diferencia entre tareas}

En las secciones previas vimos que, a excepción del último punto discutido arriba, las poblaciones de niños y adultos se comportan de manera análoga en relación con las tendencias de la EAP en las dos tareas de elicitación. Sin embargo, también notamos algunas diferencias importantes entre ambas tareas. En relación con el tipo de codificación de los referentes, habíamos apuntado arriba (§3.1, Cuadro 5) que en el corpus de cuentos aparecía, en ambas poblaciones por igual, un número alto de cláusulas transitivas con sus dos argumentos codificados en forma léxica (33\%), en relación con el de anécdotas (en promedio 9\%). Esta tendencia se confirma al analizar la distribución total de estrategias de codificación referencial en las dos tareas. Para esto, se consideraron todas las posiciones argumentales centrales directas de cada sub-corpus ( $c f$. Cuadro 3) y se distinguió entre codificación pronominal, con frases nominales definidas simples (determinante, núcleo y, opcionalmente, modificador adjetivo), con frases nominales definidas complejas (determinante, núcleo y cláusula relativa) y con 
frases nominales indefinidas. Un ejemplo de cada grupo se presenta en (9). La distribución de los datos se presenta en el Cuadro 12 para el corpus de anécdotas y en el Cuadro 13 para el corpus de cuentos.

(9) a. Él tenía tres mascotas. [Christian, cuento 6]

b. la rana no obedeció. [Fátima, cuento 3]

c. la rana que ya tenía la quiso morder. [Dania, cuento 4]

d. salieron a buscar unos juguetes para jugar. [Fátima, cuento 3]

CuADRo 12. Estrategias referenciales en el corpus de anécdotas

\begin{tabular}{|c|c|c|c|c|}
\hline \multirow{2}{*}{ Estrategia } & \multicolumn{2}{|c|}{ NIÑos } & \multicolumn{2}{|c|}{ Adultos } \\
\hline & $\#$ & $\%$ & $\#$ & $\%$ \\
\hline Pronombres & 74 & 69 & 85 & 70 \\
\hline FN definida simple & 22 & 20 & 27 & 23 \\
\hline FN definida compleja & 0 & 0 & 0 & 0 \\
\hline FN indefinida & 12 & 11 & 9 & 7 \\
\hline Total & 108 & & 121 & \\
\hline
\end{tabular}

CuAdro 13. Estrategias referenciales en el corpus de cuentos

\begin{tabular}{|c|c|c|c|c|}
\hline \multirow{2}{*}{ EstrateGIA } & \multicolumn{2}{|c|}{ Niños } & \multicolumn{2}{|c|}{ Adultos } \\
\hline & $\#$ & $\%$ & $\#$ & $\%$ \\
\hline Pronombres & 272 & 40 & 294 & 44 \\
\hline FN definida simple & 351 & 53 & 320 & 48 \\
\hline FN definida compleja & 7 & 1 & 3 & 0 \\
\hline FN indefinida & 38 & 6 & 46 & 7 \\
\hline Total & 668 & & 663 & \\
\hline
\end{tabular}

Los datos muestran que, en efecto, en el corpus de anécdotas prima para ambas poblaciones la codificación de referentes a partir de pronombres (formas cero, clíticos y tónicos), los cuales representan casi $70 \%$ de las estrategias de codificación en promedio. Por su parte, las frases definidas simples se utilizan en poco más de $20 \%$ de los contextos. No se registran frases definidas complejas, pero sí un cierto porcentaje de frases indefinidas (11\% para los niños, $7 \%$ para los adultos). En los 
cuentos, por el contrario, pronombres y frases definidas simples se distribuyen más equitativamente, con una ligera preferencia, de hecho, por el uso de estas últimas.

Respecto de estas tendencias, una hipótesis posible es que la diferencia entre los resultados de las dos tareas surge del hecho de que en el cuento aparecen más personajes que en las anécdotas personales, las cuales giran alrededor del "yo" narrador. Otra opción, que creemos es la correcta, es que el material de elicitación (el cuento en imágenes) es lo que distorsiona los datos, llevando a los hablantes a construir el relato como una secuencia de descripciones. Para distinguir más específicamente en qué contextos discursivos aparecen estas frases léxicas en los dos corpus se postularon las siguientes categorías:

1) Nuevo. Es el uso no marcado por excelencia. Se espera que los referentes discursivos nuevos se codifiquen por medio de frases nominales, independientemente de si son completamente nuevos, de si — como en los casos de mi tío, mi hermana que vimos arriba- están anclados al interlocutor y pueden ser esperables en el contexto discursivo, o de si refieren a entidades que se asumen como conocidas para el interlocutor — como el caso de el paisaje en (10). De todas maneras, estos referentes, si son nuevos en el discurso, no pueden ser referenciados de manera efectiva a través de formas pronominales:

(10) No veía el paisaje. [Antonio, cuento 5]

2) + 4 cláusulas. Este grupo reúne a los referentes codificados por última vez 4 o más cláusulas atrás. En el contexto inmediato de la mención que se analiza, el antecedente ha quedado demasiado lejos para ser recuperado a través de una mención pronominal y, por lo tanto, este es también un contexto de uso esperado para las frases nominales. En el ejemplo que se presenta en (11), es esperable que la referencia a el niño se retome en la cláusula (vi) a través de una frase nominal a pesar de su mención previa en (i). ${ }^{3}$

(11) i. Él dijo que no quería ir en una lancha.

ii. Entonces se fue la tortuga y la ranita chiquita.

3 Aquí y en lo que sigue utilizamos números romanos en lugar de letras para indicar que no se trata de una lista de ejemplos sino de una secuencia de cláusulas continuas en un fragmento narrativo. 
iii. Entonces la rana grande no quería que la ranita chiquita fuera.

iv. Entonces la rana grande la sacó

v. y la tiró al agua.

vi. Entonces el niño no quería a la rana. [Dania, cuento 4]

3) Ambiguo/contraste. En esta clase se agrupan las frases nominales que retoman un referente recién mencionado, el cual sin embargo no podría ser identificado a través de un pronombre en virtud de su competencia con otro referente con rasgos de persona, género o número similares, como se ejemplifica en (12). Es también un contexto esperado en relación con el uso de frases léxicas.

(12) i. Y con un palo se iban a ir muy lejos, pero muy muy lejos de ahí, en una madera grande, el perrito, la tortuga y la ranita chiquita.

ii. Y la ranita chiquita se empezó a reír... [Antonio, cuento 5]

4) $1 \leq 3$ cláusulas. Finalmente, este grupo representa los casos no esperados, en los que un referente recién mencionado se retoma a través de una frase nominal en ausencia de un contexto de ambigüedad, como en (13).

(13) i. Un niño recibió un día un regalo.

ii. El niño tenía un perro, una rana y una tortuga. [Romina, cuento 1]

Los resultados del análisis de los datos en función de estas categorías se presentan en el Cuadro 14 y el Cuadro 15.

Cuadro 14. Contextos de uso de frases nominales en el corpus de anécdotas

\begin{tabular}{|c|c|c|c|c|}
\hline \multirow{2}{*}{ Categoría } & \multicolumn{2}{|c|}{ Niños } & \multicolumn{2}{|c|}{ Adultos } \\
\hline & $\#$ & $\%$ & $\#$ & $\%$ \\
\hline Nuevo & 32 & 94 & 29 & 80 \\
\hline + 4 cláusulas & 2 & 6 & 6 & 17 \\
\hline Ambiguo/ contraste & -- & -- & 1 & 3 \\
\hline $1 \leq 3$ cláusulas & -- & -- & -- & -- \\
\hline Total & 34 & 100 & 36 & 100 \\
\hline
\end{tabular}


Variaciones en la realización de la estructura argumental preferida en niños y adultos 53

Cuadro 15. Contextos de uso de frases nominales en el corpus de cuentos

\begin{tabular}{|c|c|c|c|c|}
\hline \multirow{2}{*}{ Categoría } & \multicolumn{2}{|c|}{ NIÑOS } & \multicolumn{2}{|c|}{ Adultos } \\
\hline & $\#$ & $\%$ & $\#$ & $\%$ \\
\hline Nuevo & 67 & 17 & 76 & 21 \\
\hline + 4 cláusulas & 92 & 24 & 71 & 19 \\
\hline Ambiguo/ contraste & 91 & 23 & 136 & 37 \\
\hline $1 \leq 3$ cláusulas & 146 & 37 & 86 & 23 \\
\hline Total & 396 & 100 & 369 & 100 \\
\hline
\end{tabular}

En el corpus de anécdotas vemos que las frases nominales se utilizan en los contextos esperados: mayoritariamente en relación con referentes nuevos, o mencionados pero en competencia con otros. No aparece ningún caso, en este corpus, de frases nominales retomando un referente mencionado en el contexto discursivo inmediato. La distribución es muy diferente en el corpus de cuentos. Aquí 23\% de las frases nominales utilizadas por adultos y $37 \%$ de las utilizadas por niños retoman un referente que acaba de ser mencionado. En efecto, entonces, se comprueba que la predominancia relativa de frases nominales en el corpus de cuentos no resulta de la introducción de nuevos participantes, ni siquiera de modo absoluto de la necesidad de resolver contextos de ambigüedad referencial sino, de modo importante, de su uso en contextos no esperados, en los que se retoma un "tópico continuo" (Givón, 1983). En la medida en que este corpus se obtiene a partir de cuentos en imágenes, estos resultados sugieren que los hablantes $-\mathrm{y}$ los niños en mayor medida que los adultos - no están construyendo el relato como una narrativa, sino como una secuencia de descripciones de las distintas láminas.

\section{Comentarios Finales}

Este estudio tuvo como objetivo examinar en qué medida el desarrollo de capacidades discursivas de niños de 8 años se refleja también, y de modo análogo al que ha sido estudiado en otros dominios, en las tendencias comprobadas para el discurso adulto en términos de las realizaciones preferidas de la estructura argumental. Los datos han mostrado que, en efecto, los niños de 8 años que participaron en el estudio han incorporado en sus discursos las estrategias pragmáticas 
previstas por el modelo de la EAP, prefiriendo construir cláusulas transitivas con un argumento léxico o nuevo como máximo y evitando la introducción de participantes nuevos, codificados con formas léxicas, en el rol de sujeto transitivo.

Por otra parte, del análisis de los datos también surgen algunas diferencias, menores, entre las poblaciones. En particular, vimos que en el corpus de anécdotas del discurso infantil se observa, comparado con el discurso adulto, una mayor frecuencia de participantes nuevos en el rol de sujeto transitivo. Sin embargo, vimos que esta desviación respecto del discurso adulto no resulta de factores ligados al desarrollo de competencias discursivas. Por el contrario, se explica desde la experiencia vital del niño y por el tipo de material con el que construye sus anécdotas personales, involucrando centralmente a los integrantes de su círculo familiar y ubicándolos como AGENTES de los eventos narrados.

Finalmente, observamos que las diferencias más importantes surgen de la comparación entre tareas y no entre poblaciones. En efecto, mientras que en las anécdotas los referentes son codificados predominantemente a través de formas pronominales (Cuadro 12), en el corpus de cuentos los pronombres alternan con frases nominales de modo casi equivalente (Cuadro 13), algo que ya sugerían los resultados en términos de la EAP. En este sentido, el análisis más detallado de los contextos de uso de las frases nominales mostró que, en los cuentos, estas aparecen con frecuencia asociadas a contextos, no esperados, de alta continuidad tópica (i.e. denotando referentes mencionados en el contexto inmediato, recuérdese el ejemplo de (13)). Vimos que esta tendencia es particularmente notable en el discurso infantil, en el que estos usos representan $37 \%$ de los contextos de aparición de frases nominales (Cuadro 15), y sugiere que los niños tienen mayor dificultad que los adultos para construir un discurso narrativo a partir de la consigna de narrar un cuento en imágenes.

Queda pendiente analizar en qué medida el uso de frases nominales para la codificación de tópicos continuos coincide particularmente con el cambio entre una y otra lámina en el cuento en imágenes, dando lugar a un efecto de "quiebre" atencional similar al reportado por Tomlin (1987). Más allá de esta limitante, sin embargo, esperamos que este estudio aporte evidencias adicionales que contribuyan, en particular, a nuestro conocimiento del desarrollo de las competencias pragmáticas en los niños y, en general, al funcionamiento de la interfaz sintaxis-pragmática. 
Variaciones en la realización de la estructura argumental preferida en niños y adultos 55

\section{REFERENCIAS}

Aguilar, CÉsAr A. (2003). Análisis de frecuencia de construcciones anafóricas en narraciones infantiles. Estudios de Lingüística Aplicada, 38, 33-43.

Allen, Shanley E. M., \& Schröder, Heike (2003). Preferred argument structure in early Inuktitut spontaneous speech data. En John W. Du Bois, Lorraine E. Kumpf \& William J. Ashby (Eds.), Preferred argument structure: Grammar as architecture for function (pp. 301-338). Ámsterdam: John Benjamins.

Amaro, María Elena (2010). Estrategias lingüísticas para el manejo de información nueva y dada en el discurso narrativo infantil (Tesis de licenciatura inédita). Universidad Veracruzana, Xalapa.

Ashby, William J., \& Bentivoglio, Paola (1993). Preferred Argument Structure in spoken French and Spanish. Language variation and change, 5(1), 61-76.

Barriga Villanueva, Rebeca (2002). Estudios sobre habla infantil en los años escolares: un solecito calientote. México: El Colegio de México.

Barriga Villanueva, Rebeca (Ed.) (2014). Las narrativas y su impacto en el desarrollo lingüístico infantil. México: El Colegio de México. (Pról. Rebeca Barriga Villanueva)

Belloro, Valeria A., \& Guerrero, Lilian Graciela (2012). La interfaz gramática-discurso en las construcciones de movimiento en yaqui. Lingüística, 27(1), 197-220.

Bentivoglio, Paola (1983). Topic continuity and discontinuity in discourse: A study of spoken Latin-American Spanish. En Thomas Givón (Ed.), Topic continuity in discourse. A quantitative cross-language study (pp. 255-311). Ámsterdam: John Benjamins.

Bentivoglio, Paola (1992). La estructura argumental preferida en el español moderno. En María Vaquero \& Amparo Morales (Eds.), Homenaje a Humberto López Morales (pp. 107-120). Madrid: Arco Libros.

Bentivoglio, Paola (1994). Spanish Preferred Argument Structure across time and space. Revista de Documentação de Estudos em Linguistica Teórica e Aplicada (DELTA), 10, 227-293.

Bentivoglio, Paola (julio, 1996). Acquisition of Preferred Argument Structure in Venezuela Spanish. Presentación en el $7^{\circ}$ Congreso Internacional para el Estudio del Lenguaje Infantil, Estambul, Turquía.

Berman, Ruth A., \& Slobin, Dan Isaac (1994). Relating events in narrative: A crosslinguistic developmental study. Nueva York: Lawrence Erlbaum.

Clancy, Patricia (1993). Preferred Argument Structure in Korean acquisition. En Eve V. Clark (Ed.), Proceedings of the $25^{\text {th }}$ Annual Child Language Research Forum (pp. 307-314). Stanford: Center for the Study of Language and Information Publications. 
Clancy, Patricia (1995). Subject and object in Korean acquisition: Surface expression and case-marking. En Susumu Kuno, Ik-Hwan Lee, John Whitman, Joan Maling, Young-Se Kang \& Young-joo Kim (Eds.), Harvard Studies in Korean Linguistics VI (pp. 3-17). Seúl: Hanshin.

Clancy, Patricia (1996). Referential strategies and the co-construction of argument structure in Korean acquisition. En Barbara Fox (Ed.), Studies in anaphora (pp. 33-68). Ámsterdam: John Benjamins.

Clancy, Patricia (1997). Discourse motivations of referential choice in Korean acquisition. En Ho-min Sohn \& John Haig (Eds.), Japanese/Korean Linguistics, 6 (pp. 639-659). Stanford: Center for the Study of Language and Information Publications.

Clancy, Patricia (2003). The lexicon in interaction: Developmental origins of Preferred Argument Structure. En John W. Du Bois, Lorraine Edith Kumpf \& William J. Ashby (Eds.), Preferred Argument Structure: Grammar as architecture for function (pp. 81-108). Ámsterdam: John Benjamins.

Du BoIs, John W. (1987). The discourse basis of ergativity. Language, 63(4), 805-855.

Du Bois, John W.; Kumpf, Lorraine Edith, \& Ashby, William J. (2003). Preferred Argument Structure. Grammar as architecture for function. Ámsterdam: John Benjamins.

Givón, Thomas (Ed.) (1983). Topic continuity in discourse. A quantitative cross-language study. Ámsterdam: John Benjamins.

Hess Zimmermann, KARINA (2010). Saber lengua: lenguaje y metalenguaje en los años escolares. México: El Colegio de México.

Hess Zimmermann, Karina, \& Auza Benavides, Alejandra (Eds.) (2013). ¿Qué me cuentas? Narraciones y desarrollo lingüístico en niños hispanohablantes. México: Universidad Autónoma de Querétaro/Hospital General Dr. Manuel Gea González / Ediciones DeLaurel.

Khorounjaia, Ekaterina, \& Tolchinsky, Liliana (2004). Discursive constraints on the lexical realization of arguments in Spanish. En Ruth A. Berman (Ed.), Language development across childhood and adolescence (pp. 83-109). Ámsterdam: John Benjamins.

Kupersmitt, Judy, \& Berman, Ruth A. (2001). Linguistic features of Spanish-Hebrew children's narratives. En Ludo Verhoeven \& Sven Strömqvist (Eds.), Narrative development in a multilingual context (pp. 277-317). Ámsterdam: John Benjamins.

Labov, William (1972). The transformation of experience in narrative syntax. En William Labov (Ed.), Language in the inner city: Studies in Black English vernacular (pp. 354-396). Filadelfia: University of Washington Press.

Mayer, Mercer, \& Mayer, Marianna (1977). One frog too many. Nueva York: Dial Press.

Romero Contreras, Silvia, \& Gómez Martínez, Gloria Elena (2013). Exploraciones en torno a la construcción del discurso narrativo en niños de 3 a 12 años. En Karina Hess Zimmermann \& Alejandra Auza Benavides (Eds.), ¿Qué me cuentas? Narraciones y desarrollo lin- 
Variaciones en la realización de la estructura argumental preferida en niños y adultos 57

güístico en niños hispanohablantes (pp. 25-58). México: Universidad Autónoma de Querétaro / Hospital General Dr. Manuel Gea González / Ediciones DeLaurel.

Rubino, CARL (1996). The introduction of new information and Preferred Argument Structure in Central Alaskan Yup'ik narratives. En M. Mithun (Ed.), Santa Barbara papers in Linguistics. Volume 7: Prosody, grammar, and discourse in Central Alaskan Yup'ik (pp. 139-153). Santa Barbara: Linguistics Department, University of California at Santa Barbara.

Shiro, Martha (1999). Echar el cuento: hacia un perfil de las destrezas narrativas orales en niños caraqueños. Lenguas modernas, 26-27, 135-167.

Shiro, Martha (2007). El discurso narrativo oral en la vida cotidiana: géneros y proceso. En Adriana Bolívar (Ed.), Análisis del discurso. ¿Por qué y para qué? (pp. 121-133). Caracas: Los libros de El Nacional.

Shiro, Martha (2012). Y entonces le dijo... la representación del habla en las narraciones de niños venezolanos. Boletín de Lingüística, XXIV(37-38), 119-143.

Tomlin, Russell (1987). Linguistic reflections of cognitive events. En Russell Tomlin (Ed.), Coherence and grounding in discourse (pp. 455-479). Ámsterdam: John Benjamins. 
